\title{
A Novel Automated Approach to Serial Block Face DualBeam Electron Micros- copy for the Exploration of Cortical Circuits
}

\author{
G.W. Knott, ${ }^{*}$ H. Mulders, ${ }^{*}$ D.C. Wall, $* *$ D.B. Chklovskii, $* * *$ S. Reyntjens, ${ }^{* *}$ B.H. Lich, ${ }^{* *}$ \\ * DBCM, Faculté de Médecine, Université de Lausanne, Switzerland CH1005 \\ ** FEI Company, Achtsteweg Noord 5, 5600 KA Eindhoven, The Netherlands \\ ***HHMI, Cold Spring Harbor Labs, New York 11724, USA
}

The mammalian brain comprises interconnected neurons that communicate via synapses to form a bewilderingly complex network. In every micro-liter of cortical grey matter there are approximately one billion synapses arranged along several kilometers of axons. Visualizing these circuits and all their connections is paramount to being able to understand how the brain works. To achieve this would require an imaging technique that has both the resolution to distinguish the different types of synaptic connections as well as a wide enough field of view to identify all of them.

Synapses are specialized junctions whose sizes range from over a micron in diameter to less than $100 \mathrm{~nm}$. On the presynaptic side, membrane-bound vesicles of around 30nm in size cluster around a thickened membrane, or active zone, that lies opposite the postsynaptic density of the other cell. The size of these features means that synapses can only be identified using the electron microscope, and aspects of their arrangement allow them to be classified according to their function, ie. inhibitory or excitatory. Therefore, to understand the connectivity between neurons in the cortex, a meaningful volume of neural tissue needs to be imaged at 'synaptic resolution' and in three dimensions (3d).

For many years serial section transmission electron microscopy has been the only method available. Typically, fixed, stained and embedded tissue samples are sectioned with an ultra-microtome at 50-100 nm thickness into ribbons of serial sections that are aligned onto grids. These grids are then imaged in the microscope yielding stacks of images representing a $3 \mathrm{~d}$ volume in which the neuronal elements can be reconstructed and analyzed. However, this is time consuming and technically demanding work. Each section needs to being collected by hand, and long series of images can often be interrupted by section folds and beam damage.

A recent study has explored an alternative method for serial image acquisition in which the block face of resin-embedded neural tissue was imaged within a field emission gun scanning electron microscope (1). Sections were removed from the imaged face in the microscope using an ultra microtome. This provides a series of aligned images of the tissue in the block face and has the clear advantage that image acquisition can be fully automated.

We have explored a different approach of in situ sectioning by using a focused ion beam, directed parallel to the block face, for cutting away the resin. This method is referred to as Slice and View (2), and has the advantages of consistent sectioning intervals, to as low as $40 \mathrm{~nm}$, and removing the risk of mechanical damage to the block face caused by a knife. A resin embedded block of adult mouse neocortex was mounted within the microscope and images from the block face acquired with a solid state backscatter detector. Serial images were collected from the same region at steps of $40 \mathrm{~nm}$ intervals. 
Results show that this focused ion beam technique is capable of sectioning large areas of neural tissue, and using the back scattered electrons we were able to visualize the detailed ultrastructure. Synaptic contacts were clearly visible, including the pre- and post synaptic densities and synaptic vesicles. In a fully automated mode serial images were collected that allowed us to make detailed morphological analyses of the neuronal elements in 3d (Figure 3).

\section{References}

1. Denk W, Horstmann H (2004) Serial block-face scanning electron microscopy to reconstruct three-dimensional tissue nanostructure. PLoS Biol 2:e329.

2. Drobne D, Milani M, Zrimec A, Leser V, Berden Zrimec M (2005) Electron and ion imaging of gland cells using the FIB/SEM system. J Microsc 219:29-35.

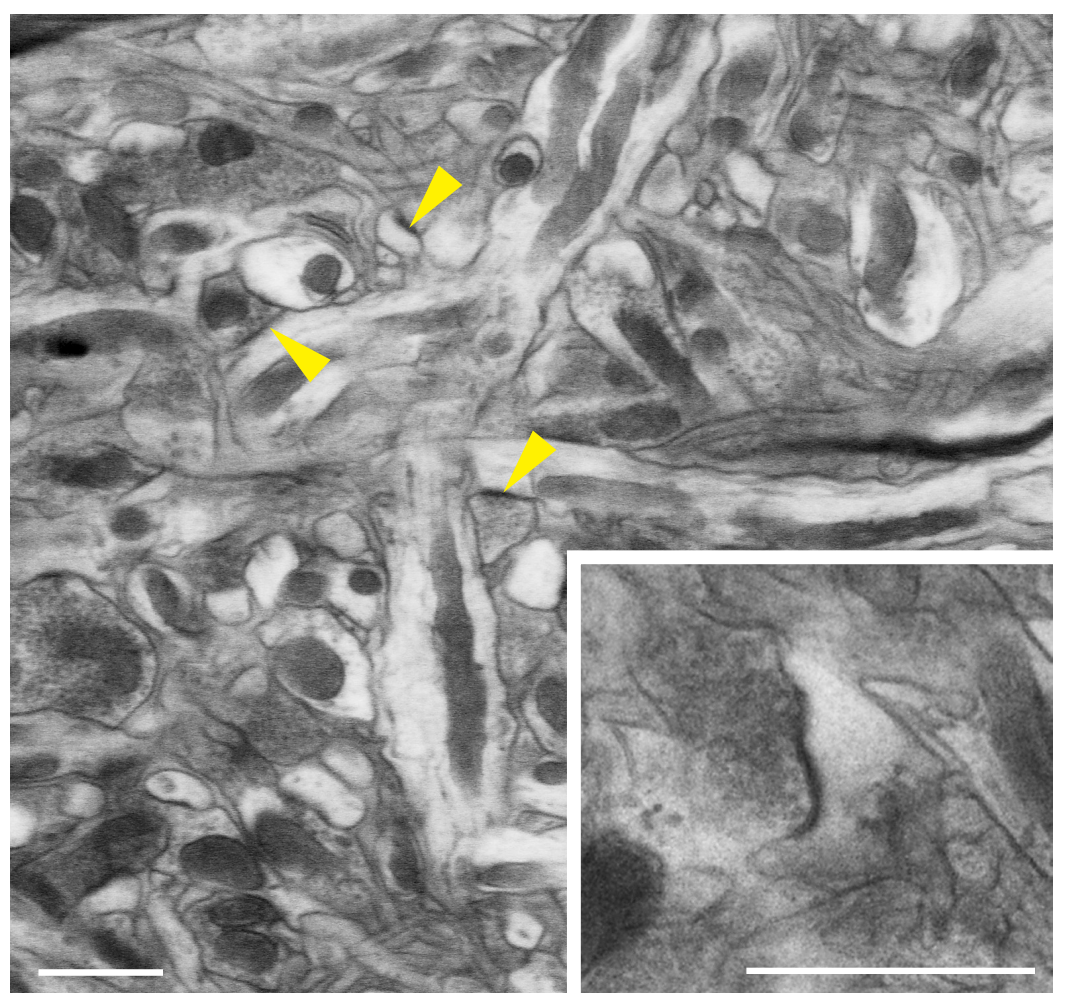

Figure 1 (left). Low power scanning EM image (inverted contrast) of the block face showing the neuronal ultrastucture. Clearly visible are synapses (yellow arrowheads), shown at a higher magnification in the inset. Scale bars are 1 micron.

Figure 3 (right). Three dimensional reconstruction of a dendrite made from serial images scanned from the block face. This contains a mitochondria (green) and protruding from the dendritic shaft is a spine that synapses (red) with an axonal bouton (blue). 